\title{
In Vivo Tumor-Targeted Dual-Modality PET/Optical Imaging with a Yolk/Shell-Structured Silica Nanosystem
}

\author{
Sixiang Shi ${ }^{1} \cdot$ Feng Chen $^{2} \cdot$ Shreya Goel $^{1} \cdot$ Stephen A. Graves $^{3} \cdot$ Haiming Luo $^{2}$ - Charles P. Theuer ${ }^{4}$ \\ Jonathan W. Engle ${ }^{3} \cdot$ Weibo Cai ${ }^{1,2,3,5}$
}

Received: 23 April 2018/Accepted: 14 June 2018/Published online: 16 July 2018

(C) The Author(s) 2018

\section{Highlights}

- A hybrid yolk/shell nanosystem was generated with quantum dot as the core and hollow mesoporous silica as the shell.

- Dual-modality PET/optical imaging was conducted to achieve synergistic cancer diagnosis that combines the advantages of both PET and optical imaging.

- Successful tumor vasculature targeting was achieved, which significantly enhanced tumor retention and targeting specificity.

\begin{abstract}
Silica nanoparticles have been one of the most promising nanosystems for biomedical applications due to their facile surface chemistry and non-toxic nature. However, it is still challenging to effectively deliver them into tumor sites and noninvasively visualize their in vivo biodistribution with excellent sensitivity and accuracy for effective cancer diagnosis. In this study, we design a yolk/ shell-structured silica nanosystem ${ }^{64} \mathrm{Cu}-\mathrm{NOTA}$ QD@HMSN-PEG-TRC105, which can be employed for tumor vasculature targeting and dual-modality PET/optical imaging, leading to superior targeting specificity, excellent
\end{abstract}

Sixiang Shi and Feng Chen have contributed equally to this work.

Weibo Cai

wcai@uwhealth.org

1 Department of Materials Science and Engineering, University of Wisconsin-Madison, Madison, WI, USA

2 Department of Radiology, University of Wisconsin-Madison, Madison, WI 53705-2275, USA

3 Department of Medical Physics, University of WisconsinMadison, Madison, WI, USA

4 TRACON Pharmaceuticals, Inc., San Diego, CA, USA

5 University of Wisconsin Carbone Cancer Center, Madison, WI, USA
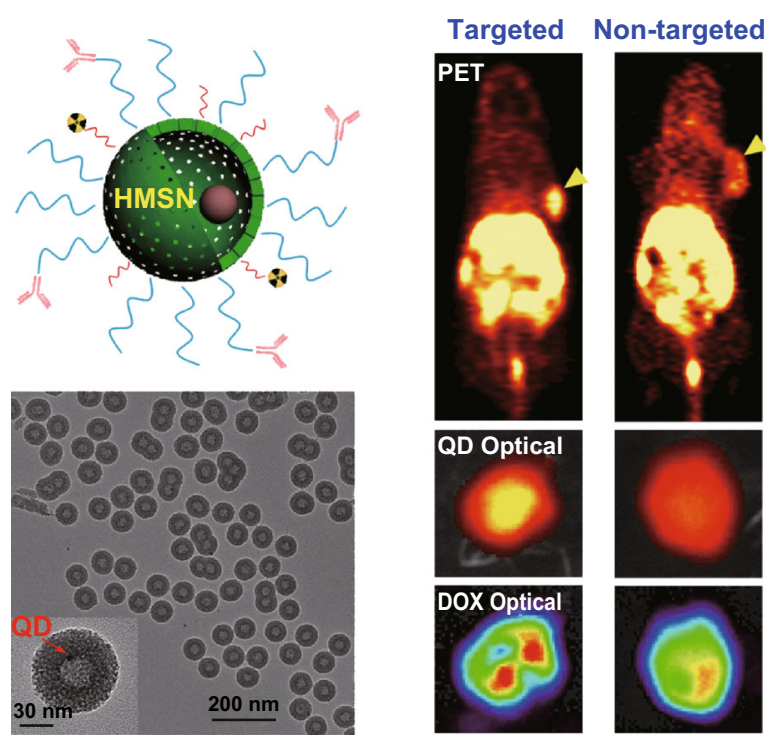

imaging capability and more reliable diagnostic outcomes. By combining vasculature targeting, $\mathrm{pH}$-sensitive drug delivery, and dual-modality imaging into a single platform, as-designed yolk/shell-structured silica nanosystems may be employed for the future image-guided tumor-targeted drug delivery, to further enable cancer theranostics. 
Keywords Hollow mesoporous silica nanoparticle (HMSN) · Quantum dot (QD) · Molecular imaging · Positron emission tomography (PET) · Optical imaging · CD105/endoglin

\section{Introduction}

In the last decade, rapid development of nanotechnology has dramatically stimulated the progress in design and synthesis of multifunctional nanosystems that can potentially be used for cancer-targeted imaging and therapy [1-5]. Among them, silica nanoparticles (NPs) have been one of the most widely studied nanosystems, due to their facile surface chemistry and non-toxic nature [6-8]. Silicabased NPs are "generally recognized as safe" by the United States Food and Drug Administration (FDA), highlighting their great clinical relevance [6-8].

Hollow mesoporous silica NPs (HMSNs), which possess numerous nanoscale pores and a large cavity inside the silica shell, have been recently developed for drug delivery due to their low mass density, large surface area, high pore volume, and uniform and tunable pore size [8-10]. Tumor active targeting has been applied to HMSNs in vivo to significantly enhance the tumor uptake and reduce possible side effects, leading to a desirable nanoplatform for cancer treatment [11-13]. Positron emission tomography (PET) has been accomplished with radiolabeled HMSNs to invasively understand their biodistribution in living animals [11-13]. Compared to traditional nanosystems, radiolabeled actively targeted HMSNs combine cancer diagnosis and therapy into one single platform, making cancer theranostics possible.

However, one imaging modality is not enough to accurately represent the in vivo fate of HMSNs [14-16]. Although PET imaging is highly quantitative and sensitive and has unlimited tissue penetration, it can only depict the in vivo biodistribution of radioisotopes, whether they are attached to or free from the carrier NPs [17-20]. Therefore, PET imaging alone may possibly lead to a totally different readout, owing to different biodistribution profiles of NP agents and free radioisotopes, which results in a false diagnosis. To overcome this drawback, we have designed a quantum dot (QD)/HMSN yolk/shell nanosystem (QD@HMSN) that makes use of the optical properties of QDs for fluorescence imaging. Compared to other fluorescent emitters such as organic dyes, QDs have wider excitation and narrower emission spectra, higher quantum yields and minimal photo bleaching, and therefore can accurately render the biodistribution of HMSNs, providing synergistic diagnostic information in addition to PET [21]. The incorporated QDs were placed in the cavity and protected by the silica shells, which will not alter the intrinsic pharmacokinetics of HMSNs in the living systems.

Unlike small molecules or antibody, NPs have a relatively large size and exhibit suboptimal extravasation from vessels, which limits their applications in tumor cell targeting. Tumor vasculature targeting avoids the need of extravasation and has become one of the most effective active targeting methods for NPs [22]. Endoglin receptor (CD105) is a type I membrane glycoprotein, which highly proliferates in tumor neovasculature and plays an important role in tumor angiogenesis, growth and metastasis [23-26]. In this study, a chimeric human/murine anti-CD105 antibody, TRC105, was conjugated onto the surface of QD@HMSN yolk/shell nanosystems for effective tumor vasculature targeting and enhanced tumor diagnosis.

\section{Materials and Methods}

\subsection{Materials}

Chelex 100 resin (50-100 mesh), tetraethyl orthosilicate (TEOS), triethanolamine (TEA), (3-aminopropyl)triethoxysilane (APTES), and cetyltrimethylammonium chloride solution (CTAC, $25 \mathrm{wt} \%$ ) were purchased from Sigma-Aldrich (St. Louis, MO). TRC105 was provided by TRACON Pharmaceuticals Inc. (San Diego, CA). Complete mouse serum was purchased from Jackson Immuno Research Laboratories (West Grove, PA). 1,4,7-Triazacyclononane-1,4,7-triacetic acid (NOTA) was purchased from Macrocyclics, Inc. (Dallas, TX). ${ }^{64} \mathrm{Cu}$ was produced by a GE PETtrace cyclotron using the ${ }^{64} \mathrm{Ni}(\mathrm{p}, \mathrm{n}){ }^{64} \mathrm{Cu}$ reaction. Water and all buffers were of Millipore grade and pre-treated with Chelex 100 resin to ensure that the aqueous solution was free of heavy metal. All other chemicals and buffers were obtained from Thermo Fisher Scientific (Fair Lawn, NJ).

\subsection{Synthesis of QD@HMSN Yolk/Shell Nanosystems}

QD NPs (Qdot ${ }^{\mathrm{TM}} 705$ ITK $^{\mathrm{TM}}$ carboxyl quantum dots, Thermo Fisher Scientific, Fair Lawn, NJ) were first coated with dense silica $\left(\mathrm{dSiO}_{2}\right)$ layer, by oil-in-water reverse micro-emulsion silica coating approach. In brief, Igepal CO-520 (NP-5, $1 \mathrm{~mL}$ ) was dispersed in cyclohexane $(20 \mathrm{~mL})$ in a $100-\mathrm{mL}$ three-necked flask and stirred at a slow rate for $5 \mathrm{~min}$. Qdot705 (ITK organic quantum dots, $400 \mathrm{pmol}$ ) was added into $\mathrm{CO}-520$ solution and stirred slowly for $2 \mathrm{~h}$. Ammonia solution $(0.14 \mathrm{~mL}, 30 \%)$ was then added into the mixture and stirred for another $2 \mathrm{~h}$. 
TEOS was subsequently pumped into the reaction $\left(100 \mu \mathrm{L} \mathrm{h}^{-1}\right)$ for $2 \mathrm{~h}$ and stirred at room temperature for $40 \mathrm{~h}$. When the reaction was completed, $1 \mathrm{~mL}$ methanol and $2 \mathrm{~mL}$ ethanol were added into the solution to precipitate the sample. The QD@ $\mathrm{dSiO}_{2}$ samples were collected by centrifugation and washed with ethanol for three times.

As-synthesized QD@ $\mathrm{dSiO}_{2}$ NPs were further coated with mesoporous silica NPs (MSNs) to form the mesoporous silica shell. In brief, $20 \mu \mathrm{g}$ TEA and $10 \mathrm{~mL}$ CTAC (25 wt $\%$ ) were dissolved into $20 \mathrm{~mL}$ water and stirred for $1.5 \mathrm{~h}$. Ten milliliters of $\mathrm{QD} @ \mathrm{dSiO}_{2}$ solution (containing 200 pmol QDs) was then added and stirred for another $1.5 \mathrm{~h}$. TEOS was subsequently added at the rate of $20 \mu \mathrm{L} \mathrm{min}{ }^{-1}$ for $5 \mathrm{~min}$. The reaction took place at $80{ }^{\circ} \mathrm{C}$ for $1 \mathrm{~h}$. After the reaction was finished, QD@ $\mathrm{dSiO}_{2} @ \mathrm{MSN}$ solution was mixed with $636 \mathrm{mg} \mathrm{Na}_{2} \mathrm{CO}_{3}$ and moved to $50{ }^{\circ} \mathrm{C}$ water bath for $45 \mathrm{~min}$ to etch out $\mathrm{dSiO}_{2}$. The resulting QD@HMSN NPs were collected by centrifugation and washed with water for three times. To remove CTAC, the product was extracted for $24 \mathrm{~h}$ with a $1 \mathrm{wt} \%$ solution of $\mathrm{NaCl}$ in methanol at room temperature. This process was carried out for at least three times to ensure the complete removal of CTAC.

\subsection{Surface Engineering of QD@HMSN Yolk/Shell Nanosystems}

$\mathrm{NH}_{2}$ groups were introduced onto the surface of QD@HMSN NPs for further surface engineering. QD@HMSN NPs were dispersed in $20 \mathrm{~mL}$ of absolute ethanol, followed by the addition of $1 \mathrm{~mL}$ of APTES. The system was sealed and kept at $86-90{ }^{\circ} \mathrm{C}$ for reaction for $24 \mathrm{~h}$. When the reaction was completed, the $\mathrm{NH}_{2}$-modified QD@HMSN (QD@HMSN-NH N $_{2}$ was collected by centrifugation and washed with absolute ethanol for three times to remove the remaining APTES.

For chelating radioisotopes, p-SCN-Bn-NOTA was conjugated onto QD@HMSN NPs based on the reaction between $\mathrm{SCN}$ and $\mathrm{NH}_{2}$ groups at $\mathrm{pH} 9$ for $2 \mathrm{~h}$ (QD/NOTA molar ratio 1:500; of note, since QD is the seed of HMSN, the molar concentrations of QD and HMSN NPs were nearly identical). Fluorescein isothiocyanate (FITC) was conjugated onto QD@HMSN NPs with the same method for in vitro experiments. The resulting NOTA-QD@HMSN or FITC@HMSN was then conjugated with $\mathrm{SCM}-\mathrm{PEG}_{5 \mathrm{k}}-\mathrm{Mal}$ based on the reaction between SCM group (succinimidyl carboxyl methyl ester) and remaining $\mathrm{NH}_{2}$ groups at $\mathrm{pH} 7$ for $2 \mathrm{~h}$ (QD/PEG molar ratio 1:10,000). Meanwhile, TRC105 (QD/TRC105 molar ratio 1:20) was reacted with Traut's reagent (TRC105/ Traut's reagent molar ratio $1: 20$ ) at $\mathrm{pH} 8$ for $1 \mathrm{~h}$ to introduce free $\mathrm{SH}$ groups on the antibodies. Finally, as-prepared NOTAQD@HMSN-PEG or FITC-QD@HMSN-PEG was mixed with TRC105-SH and reacted at $\mathrm{pH} 7.4$ for $2 \mathrm{~h}$ in the presence of tris(2-carboxyethyl)phosphine (TCEP, to prevent oxidation of the thiol), generating the final products NOTAQD@HMSN-PEG-TRC105 or FITC-QD@HMSN-PEGTRC105 (Fig. 1).

\subsection{Cell Lines and Animal Models}

All animal studies were conducted under a protocol approved by the University of Wisconsin Institutional Animal Care and Use Committee. 4T1 murine breast cancer cells were obtained from American Type Culture Collection (ATCC, Manassas, VA) and cultured according to the supplier's instructions. When they reached $\sim 80 \%$ confluence, the cells were harvested for tumor implantation. Four-to-five-week-old female Balb/c mice (Envigo, Indianapolis, IN) were each subcutaneously injected with $2 \times 10^{6} 4 \mathrm{~T} 1$ cells in the flank to generate the $4 \mathrm{~T} 1$ breast cancer model. The mice were used for in vivo experiments when the tumor diameter reached 6-8 $\mathrm{mm}$.

\subsection{In Vitro CD105 Targeting}

Human umbilical vein endothelial cells (HUVECs, CD105 positive) were harvested and suspended in cold PBS with $2 \%$ bovine serum albumin at a concentration of $5 \times 10^{6}$ cells $\mathrm{mL}^{-1}$, incubated with FITC-QD@HMSNPEG or FITC-QD@HMSN-PEG-TRC105 at a concentration of $10 \mathrm{pmol} \mathrm{mL}^{-1}$ (based on QD concentration) for $30 \mathrm{~min}$ at room temperature. A blocking experiment (500 $\mu \mathrm{g} \mathrm{mL}^{-1}$ of TRC105 added to the cells $3 \mathrm{~h}$ before administration of NPs) was also carried out to further validate the targeting specificity. Afterward, the cells were collected by centrifugation at $1000 \mathrm{rpm}$ for $5 \mathrm{~min}$ and washed three times with cold PBS. The cells were analyzed using a BD FACSCalibur 4-color analysis cytometer equipped with 488 and $633 \mathrm{~nm}$ lasers (Becton-Dickinson, San Jose, CA) and FlowJo analysis software (Tree Star, Inc., Ashland, OR).

\subsection{Radiolabeling and In Vivo PET Imaging}

${ }^{64} \mathrm{CuCl}_{2}$ (74 MBq) was diluted in $300 \mu \mathrm{L}$ of $0.1 \mathrm{M}$ sodium acetate buffer ( $\mathrm{pH}$ 5.5) and mixed with NOTAQD@HMSN-PEG and NOTA-QD@HMSN-PEGTRC105. The reactions were conducted at $37{ }^{\circ} \mathrm{C}$ for 45 min with constant shaking. The resulting ${ }^{64} \mathrm{Cu}-N O T A-$ QD@HMSN-PEG and ${ }^{64} \mathrm{Cu}-\mathrm{NOTA}-\mathrm{QD} @ H M S N-P E G-$ TRC105 were purified by PD-10 size exclusion column chromatography using PBS as the mobile phase. NOTAmediated ${ }^{64} \mathrm{Cu}$ labeling proved to be stable in accordance with our previous studies.

${ }^{64} \mathrm{Cu}-\mathrm{NOTA}-\mathrm{QD} @ \mathrm{HMSN}-\mathrm{PEG}$ and ${ }^{64} \mathrm{Cu}-\mathrm{NOTA}-$ QD@HMSN-PEG-TRC105 were intravenously injected to 


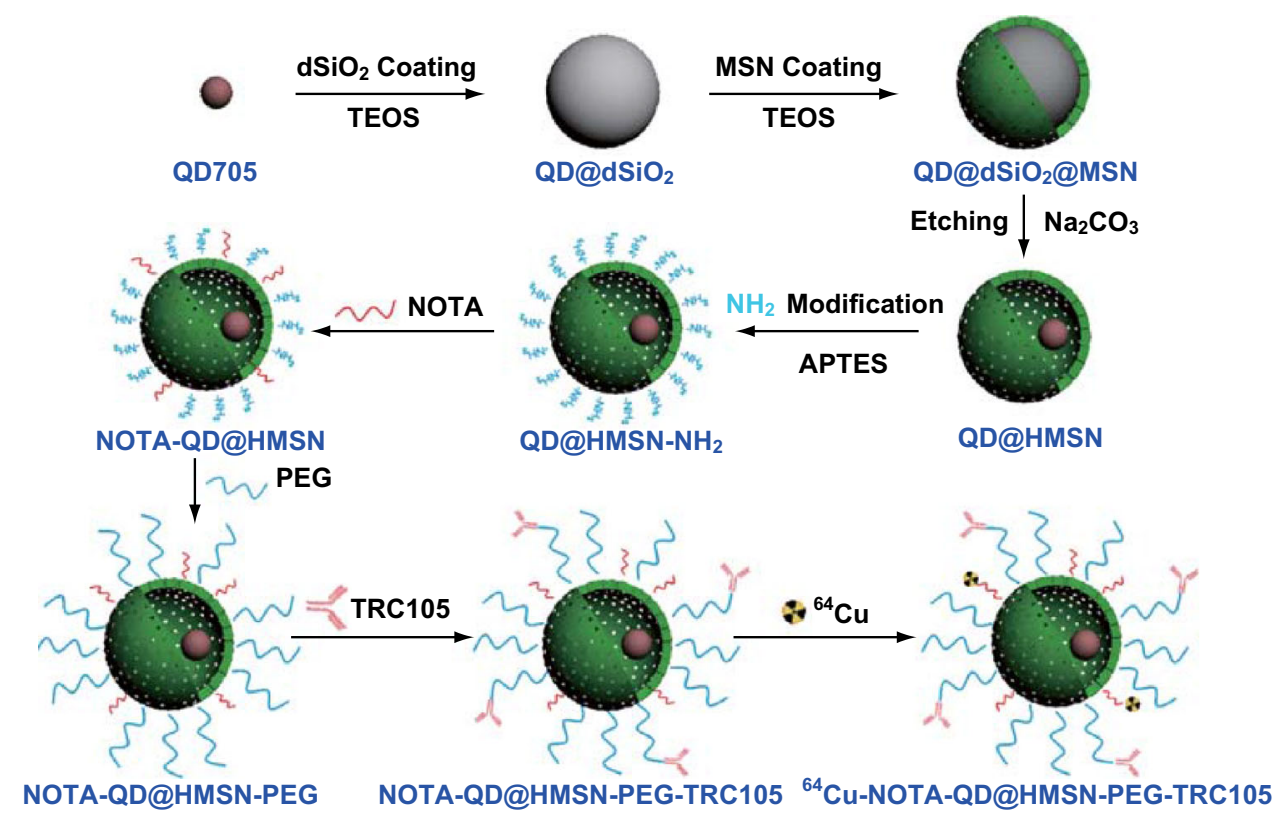

Fig. 1 A schematic illustration of the synthesis and functionalization of QD@HMSN yolk/shell-structured silica nanosystem

4T1 tumor-bearing mice. Serial PET scans were performed using a microPET/microCT Inveon rodent model scanner (Siemens Medical Solutions, USA, Inc.) at different time points $(0.5,3,6$, and $24 \mathrm{~h})$ post-injection (p.i.). Quantitative data of ROI analysis on tumor and other organs were presented as percentage injected dose per gram of tissue (\%ID/g). After the last scan at $24 \mathrm{~h}$ p.i., mice were killed under anesthesia for ex vivo biodistribution studies. Tumor, blood, and major organs/tissues were collected and weighted. The radioactivity in the tissue was measured using a $\gamma$ counter (PerkinElmer) and presented as $\% \mathrm{ID} / \mathrm{g}$ (mean $\pm \mathrm{SD}$ ).

\subsection{Drug Loading and Ex Vivo Fluorescence Imaging}

Doxorubicin (DOX) was loaded into QD@HMSN yolk/ shell nanosystems as the model drug. In brief, DOX was dissolved in DMSO $\left(10 \mathrm{mg} \mathrm{mL}^{-1}\right)$ and added into NOTAQD@HMSN-PEG or NOTA-QD@HMSN-PEG-TRC105 solutions (NP/DOX weight ratio 1:2; NP weight was measured after freeze-drying of QD@HMSN-PEG) for overnight incubation under room temperature. The DOXloaded NOTA-QD@HMSN-PEG or NOTA-QD@HMSNPEG-TRC105 (NOTA-QD@HMSN(DOX)-PEG or NOTA-QD@HMSN(DOX)-PEG-TRC105) was collected with centrifugation and washed with PBS for three times. The amount of DOX which was washed away was measured and calculated by UV-Vis absorbance at $480 \mathrm{~nm}$, based on the standard equation [washed DOX weight $=($ UV-Vis intensity-0.0356)/0.00403] which was generated from the measurement of the DOX samples with known concentrations. The final loading capacity was calculated by the equation [loading capacity $=($ mixed DOX weight washed DOX weight)/NP weight].

The drug release tests were performed by incubating DOX-loaded QD@HMSN yolk/shell nanosystems in both normal physiological environment $(\mathrm{pH}$ 7.4) and acidic environment $(\mathrm{pH} \sim 5)$. The DOX-loaded samples were centrifuged at $3,6,24$, and $48 \mathrm{~h}$ post-incubation, and the supernatants were collected and measured by UV-Vis absorbance at $480 \mathrm{~nm}$. The amount of the released DOX was calculated by the equation [released DOX weight $=($ UV-Vis intensity-0.0356)/0.00403]. The drug release rate was calculated by the equation [drug release $(\%)=$ released DOX weight/loaded DOX weight $\times 100 \%$ ].

NOTA-QD@HMSN(DOX)-PEG and NOTAQD@HMSN(DOX)-PEG-TRC105 were intravenously injected in $4 \mathrm{~T} 1$ tumor-bearing mice. After $3 \mathrm{~h}$ p.i. (when 4T1 tumor uptake was at the peak based on PET imaging), mice were killed under anesthesia. Tumor, blood, and other important organs were collected for near-infrared optical imaging using IVIS spectrum in vivo imaging system (PerkinElmer, Waltham, MA). The signals from both QD (excitation/emission wavelength $605 / 700 \mathrm{~nm}$ ) and DOX (excitation/emission wavelength $500 / 580 \mathrm{~nm}$ ) were detected and analyzed.

\subsection{Histological Analysis}

Two groups of three 4T1 tumor-bearing mice were each injected with FITC-QD@HMSN-PEG and FITC- 
QD@HMSN-PEG-TRC105 (10 pmol per mouse based on QD concentration) and euthanized at $3 \mathrm{~h}$ p.i. The $4 \mathrm{~T} 1$ tumor, liver (positive control, which has high uptake of ${ }^{64} \mathrm{Cu}-\mathrm{NOTA}-\mathrm{QD} @ \mathrm{HMSN}-\mathrm{PEG}-\mathrm{TRC} 105$ based on PET imaging), and muscle (negative control, which has low uptake of ${ }^{64} \mathrm{Cu}-\mathrm{NOTA-QD} @$ HMSN-PEG-TRC105 based on PET imaging) were frozen and cryo-sectioned for histological analysis. Frozen tissue slices with $6 \mu \mathrm{m}$ thickness were fixed with cold acetone and stained for endothelial marker CD31, as described previously using a rat antimouse CD31 antibody and a Cy3-labeled donkey anti-rat IgG. All images from both FITC (representing the location of NPs in the tissues) and Cy3 (representing the location of vasculature) channels were acquired with a Nikon Eclipse Ti microscope.

\section{Results and Discussion}

\subsection{Synthesis and Functionalization of QD@HMSN NPs}

The QD@HMSN NPs were synthesized by a two-step method, as shown in Fig. 1. The QD cores were first coated with $\mathrm{dSiO}_{2}$ through an oil-in-water reverse micro-emulsion silica coating approach [27]. This step effectively integrated hydrophobic QD cores into the silica nanosystem. By modifying the thickness of the $\mathrm{dSiO}_{2}$, we can easily control the size of the hollow cavity after etching. In the second step, as-prepared $\mathrm{QD} @ \mathrm{dSiO}_{2} \mathrm{NPs}$ were further coated with MSN shell and then selectively etched out with $\mathrm{Na}_{2} \mathrm{CO}_{3}$ to generate an inner cavity. The final size of QD@HMSN was $72 \mathrm{~nm}$, which has a large cavity ( $\sim 25 \mathrm{~nm})$ and a QD core $(\sim 5 \mathrm{~nm})$ inside each cavity, showing a distinctly different morphology compared to the QD@dSi ${ }_{2} @ M S N$ before etching (Fig. 2a). The surfactant CTAC was later removed via an extraction process by stirring the nanoparticles in $\mathrm{NaCl}$ : methanol solution (1 $\mathrm{wt} \%)$ [28]. The silica pores $(\sim 2-3 \mathrm{~nm})$ were exposed after the CTAC removal [29], which provide sufficient porous channels for efficient drug loading and release.

Amine groups $\left(\mathrm{NH}_{2}\right)$ were introduced onto the surface of QD@HMSN by reacting with APTES in absolute ethanol for further functionalization [11]. PEG chains were conjugated onto amine-modified QD@HMSN to improve the solubility in physiological solutions and biocompatibility in vitro and in vivo. NOTA was employed as the coordination chelator for radiolabeling of ${ }^{64} \mathrm{Cu}$, an excellent radioisotope for PET with the half-life of $12.7 \mathrm{~h}$. TRC105, a chimeric antibody that specifically binds to CD105, was conjugated for efficiently and specifically targeting CD105, which is exclusively expressed on the proliferating tumor vasculature. The hydrodynamic diameter of QD@HMSN was $76.3 \pm 8.9 \mathrm{~nm}$ based on dynamic light scattering, whereas that of the final conjugate NOTA-QD@HMSNPEG-TRC105 was increased to $98.1 \pm 15.9 \mathrm{~nm}$, suggesting successful conjugation of NOTA, PEG and TRC105 onto the surface of QD@HMSN.

\subsection{In Vitro CD105 Targeting}

Fluorescent-dye FITC was conjugated onto QD@HMSNPEG-TRC105 for in vitro angiogenesis targeting. As evidenced by flow cytometry results (Fig. 2b), significant enhancement was observed with the targeted group (FITCQD@HMSN-PEG-TRC105), in comparison with the negative control (PBS), non-targeted group (FITC-QD@HMSNPEG) and blocking group (FITC-QD@HMSN-PEGTRC105 administrated after injection of a large dose of TRC105 antibodies). This result indicates the successful vasculature targeting and minimal nonspecific binding of QD@HMSN-PEG-TRC105 in cell culture.

\subsection{In Vivo Vasculature Targeting and PET Imaging}

${ }^{64} \mathrm{Cu}$ was labeled onto NOTA-QD@HMSN-PEG-TRC105 (targeted group) and NOTA-QD@HMSN-PEG (non-targeted group) via simple mixing under mild conditions and purified with PD-10 desalting column. After radiolabeling, as-prepared ${ }^{64} \mathrm{Cu}-\mathrm{NOTA}-\mathrm{QD} @ H M S N-P E G-T R C 105$ (targeted group) and ${ }^{64} \mathrm{Cu}-\mathrm{NOTA}-\mathrm{QD} @ \mathrm{HMSN}-\mathrm{PEG}$ (nontargeted group) were intravenously injected into 4T1 tumor-bearing mice for in vivo vasculature targeting and PET imaging. The coronal PET images that contain the 4T1 tumors are shown in Fig. 3, and the quantitative data obtained from ROI analysis of the PET data are shown in Fig. 4.

The tumor uptake in the targeted group was prompt and persistent, manifesting as early as $3 \mathrm{~h}$ p.i. and remained visible after $24 \mathrm{~h}$ p.i. $(3.1 \pm 1.6,7.2 \pm 0.4,7.2 \pm 0.3$, and $5.6 \pm 0.3 \% \mathrm{ID} / \mathrm{g}$ at $0.5,3,6$, and $24 \mathrm{~h}$ p.i., respectively; Fig. 4a). However, significantly lower tumor uptake was observed in non-targeted $(2.7 \pm 0.5,4.6 \pm 0.2,5.0 \pm 0.6$, and $4.5 \pm 0.7 \% \mathrm{ID} / \mathrm{g}$ at $0.5,3,6$, and $24 \mathrm{~h}$ p.i., respectively; Fig. 4b) and blocking groups $(1.4 \pm 0.7,3.9 \pm 0.6$, $4.9 \pm 0.9$, and $4.7 \pm 0.7 \% \mathrm{ID} / \mathrm{g}$ at $0.5,3,6$, and $24 \mathrm{~h}$ p.i., respectively; Fig. 4c). About 1.5-fold increase in tumor uptake was achieved with the targeted group than the nontargeted and blocking groups ( $p<0.05$ at 3 and $6 \mathrm{~h}$ p.i., respectively; Fig. 4d), suggesting the excellent targeting efficiency and specificity.

To the contrary, no significant increase from targeted group was found in other normal organs. For example, most of the NPs are eventually transported to the liver and cleared via hepatobiliary pathway [22]. In this study, slight 
(a)

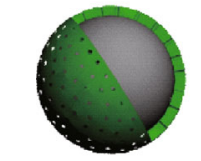

QD@dSiO ${ }_{2} @ M S N$

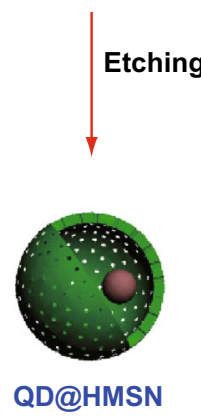

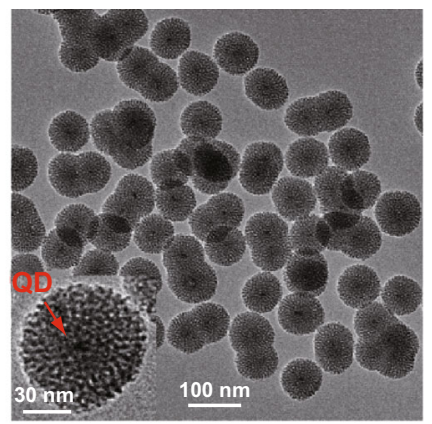

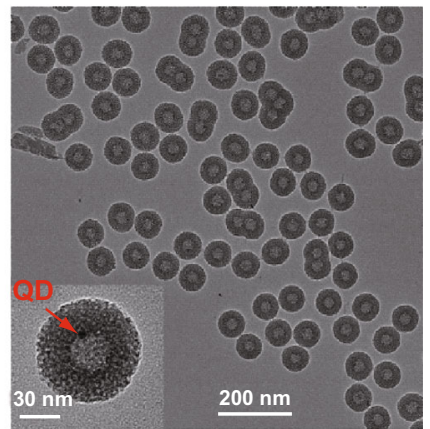

(b)

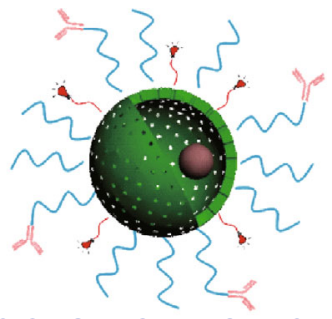

FITC-QD@HMSN-PEG-TRC105

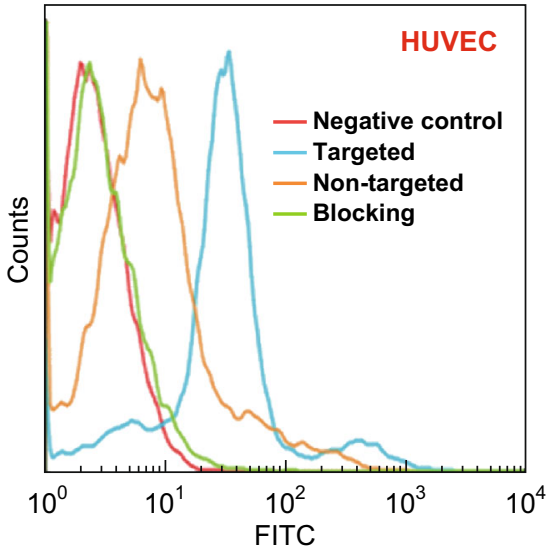

Fig. 2 a The schemes and TEM images of QD@ $\mathrm{dSiO}_{2} @ \mathrm{MSN}$ and QD@HMSN NPs. b The scheme and flow cytometry analysis of QD@HMSN yolk/shell-structured silica nanosystem in HUVECs (CD105 positive)

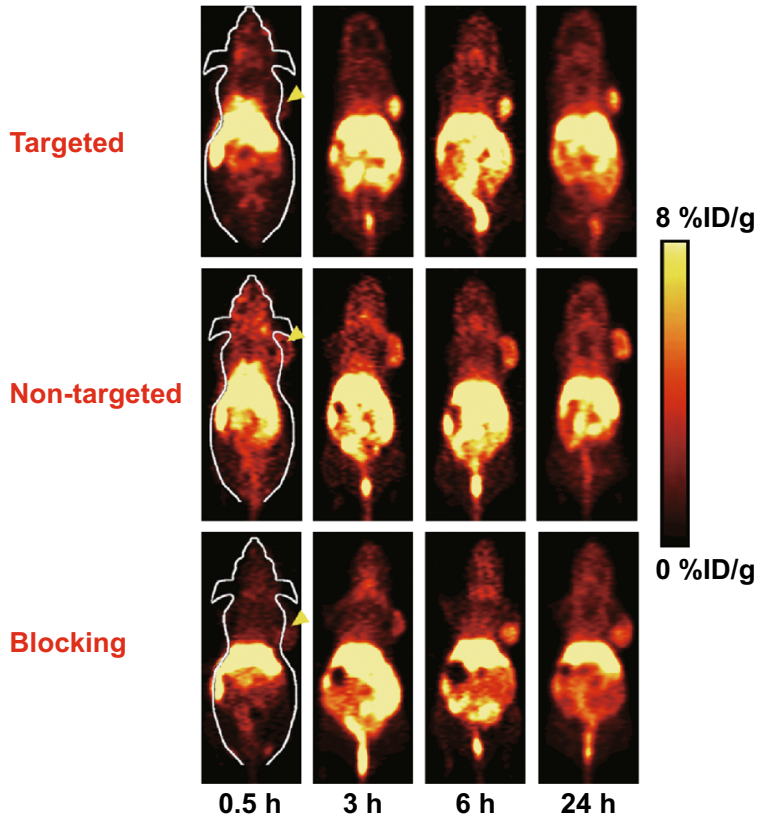

Fig. 3 Serial coronal PET images of 4T1 tumor-bearing mice at different time points post-injection of ${ }^{64} \mathrm{Cu}-\mathrm{NOTA}-\mathrm{QD} @ \mathrm{HMSN}$ PEG-TRC105 (targeted group), ${ }^{64} \mathrm{Cu}-\mathrm{NOTA}-\mathrm{QD} @ \mathrm{HMSN}-\mathrm{PEG}$ (nontargeted group), or ${ }^{64} \mathrm{Cu}$-NOTA-QD@HMSN-PEG-TRC105 after a pre-injected blocking dose of TRC105 (blocking group). Tumors are indicated by arrowheads

reduction in liver uptake was observed in the targeted group $\quad(44.2 \pm 9.8, \quad 26.3 \pm 3.0, \quad 22.7 \pm 2.8, \quad$ and $17.2 \pm 2.0 \% \mathrm{ID} / \mathrm{g}$ at $0.5,3,6$, and 24 h p.i., respectively;
Fig. 4a) than non-targeted $(43.9 \pm 4.8, \quad 32.8 \pm 4.4$, $30.1 \pm 4.4$, and $18.8 \pm 3.3 \% \mathrm{ID} / \mathrm{g}$ at $0.5,3,6$, and $24 \mathrm{~h}$ p.i., respectively; Fig. $4 \mathrm{~b})$ and blocking group ( $58.8 \pm 4.7$, $31.0 \pm 2.9,26.6 \pm 1.5$, and $20.4 \pm 0.3 \% \mathrm{ID} / \mathrm{g}$ at $0.5,3,6$, and $24 \mathrm{~h}$ p.i., respectively; Fig. 4c), possibly because more NPs were trapped in tumor tissues in the targeted groups. In addition, the muscle which has no CD105 expression exhibited nearly identical uptakes from all three groups (targeted $\quad 0.8 \pm 0.4, \quad 1.0 \pm 0.3, \quad 0.9 \pm 0.1, \quad$ and $0.7 \pm 0.1 \% \mathrm{ID} / \mathrm{g} ; \quad$ non-targeted $\quad 0.7 \pm 0.1, \quad 0.8 \pm 0.1$, $0.7 \pm 0.1$, and $0.7 \pm 0.1 \% \mathrm{ID} / \mathrm{g} ; \quad$ blocking $0.4 \pm 0.2$, $0.7 \pm 0.1,0.7 \pm 0.1$, and $0.7 \pm 0.1 \% \mathrm{ID} / \mathrm{g}$ at $0.5,3,6$, and $24 \mathrm{~h}$ p.i., respectively), suggesting minimal nonspecific binding of ${ }^{64} \mathrm{Cu}-\mathrm{NOTA}-\mathrm{QD} @ \mathrm{HMSN}-\mathrm{PEG}-\mathrm{TRC} 105$ to the normal organs. Furthermore, the blood uptakes from all three groups were also similar (targeted $6.7 \pm 2.1$, $3.3 \pm 0.1,3.3 \pm 0.3$, and $3.2 \pm 0.4 \% \mathrm{ID} / \mathrm{g}$; non-targeted $4.6 \pm 4.4, \quad 2.8 \pm 0.7, \quad 2.9 \pm 0.3$, and $2.8 \pm 0.4 \% \mathrm{ID} / \mathrm{g}$; blocking $\quad 2.9 \pm 0.6, \quad 2.9 \pm 0.1, \quad 3.2 \pm 0.1, \quad$ and $3.0 \pm 0.2 \% \mathrm{ID} / \mathrm{g}$ at $0.5,3,6$, and $24 \mathrm{~h}$ p.i., respectively), confirming the targeting specificity.

To further validate the accuracy of PET imaging, ex vivo biodistribution studies were conducted by wet weighting and measuring the radioactivities from tumor and other organs (Fig. 4e). The results corroborated well with the ROI analysis of PET images, where tumor uptake was significantly enhanced in the targeted group in comparison with the non-targeted and blocking groups. 

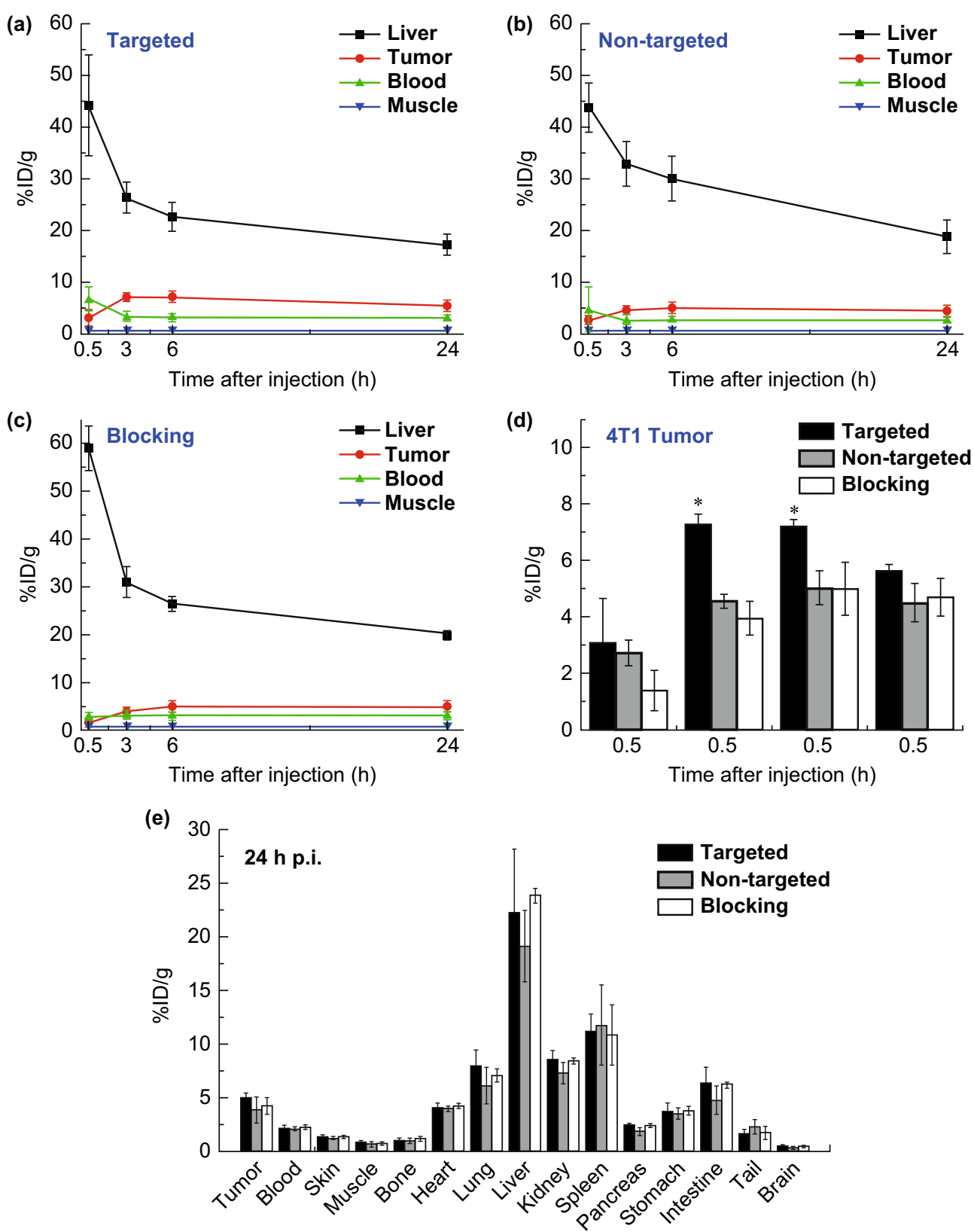

Fig. 4 a-c Time-activity curves of the liver, 4T1 tumor, blood, and muscle upon intravenous injection of ${ }^{64} \mathrm{Cu}-\mathrm{NOTA}-\mathrm{QD} @ \mathrm{HMSN}-\mathrm{PEG}-$ TRC105 (targeted group), ${ }^{64} \mathrm{Cu}-\mathrm{NOTA}-\mathrm{QD} @$ HMSN-PEG (non-targeted group), or ${ }^{64} \mathrm{Cu}-\mathrm{NOTA}-\mathrm{QD} @ \mathrm{HMSN}-\mathrm{PEG}-\mathrm{TRC} 105$ after a pre-injected blocking dose of TRC105 (blocking group). d Comparison of the 4T1 tumor uptake in the three groups. e Biodistribution studies in 4T1 tumorbearing mice at $24 \mathrm{~h}$ post-injection of the three groups of NPs. All data represent 4 mice per group $(* P<0.05)$

\subsection{Drug Loading and Optical Imaging}

One of the most important advantages of HMSN over MSN is the increased drug loading capacity. Typically, due to large surface area of MSN, the anticancer drug DOX can be easily loaded onto MSN via hydrophobic and electrostatic interactions, with a loading capacity of $\sim 400-500 \mathrm{mg} \mathrm{g}^{-1}$ (DOX weight/NP weight) [29]. After introducing a large cavity inside the MSN shells, the loading capacity was remarkably increased to
$1266 \mathrm{mg} \mathrm{g}^{-1}$ (DOX weight/NP weight) in as-designed yolk/shell QD@HMSN NPs (Fig. 5a). The increased drug loading can potentially benefit the efficacy of cancer chemotherapy [30-32] and also reduce the potential in vivo cytotoxicity from the nanocarriers since smaller dose of NPs will be needed for each treatment. In addition, the drug release rate is $\mathrm{pH}$ dependent (Fig. 5b). In normal physiological environment ( $\mathrm{pH} 7.4), 13.5 \pm 0.7 \%$ of DOX was released from QD@HMSN after 48-h incubation. However, when the $\mathrm{pH}$ decreased to $\sim 5$, drug release was 
dramatically accelerated and $34.0 \pm 0.2 \%$ of DOX was released from QD@HMSN after 48-h incubation, due to decreased hydrophobic and electrostatic interactions between DOX and silica at lower $\mathrm{pH}$ values. Since tumors generally have lower $\mathrm{pH}$ values than normal tissues, asprepared DOX-loaded QD@HMSN can be utilized as a promising $\mathrm{pH}$-sensitive drug delivery system.

Although PET imaging is highly sensitive and quantitative, it can only represent the biodistribution of radioisotopes rather than the NPs [17-19], since the detachment of radioisotopes and chelators from NPs is inevitable when they circulate in the blood stream, accumulate in livers and other organs, and interact with proteins and other biological macromolecules. Therefore, another imaging modality is recommended to confirm the accuracy of PET imaging and depict the real biodistribution of NPs. In this study, QD705 $\left(E_{x}: 605, E_{m}: 700 \mathrm{~nm}\right)$ inside each silica shell was employed for fluorescent imaging to understand the biodistribution of QD@HMSN NPs. In addition, anticancer drug DOX $\left(E_{x}: 500, E_{m}: 580 \mathrm{~nm}\right)$ that was loaded in the cavity of QD@HMSN NPs was also imaged to further validate their biodistribution. As shown in Fig. 5c, the tumors exhibited significant differences between targeted (NOTA-QD@HMSN(DOX)-PEGTRC105) and non-targeted groups (NOTAQD@HMSN(DOX)-PEG) from QD- and DOX-based optical imaging. However, no difference was found between targeted and non-targeted groups in all the normal organs. Taken together, optical imaging of QD and DOX corroborated well with the PET imaging and confirmed the successful targeting of TRC105-conjugated QD@HMSN, which can serve as a multifunctional nanoplatform for dual-modality cancer diagnosis and drug delivery.

\subsection{Histological Analysis}

Histological studies were conducted to evaluate the specificity of vasculature targeting. As shown in Fig. 6, excellent correlation was found between the signals from vasculature (CD 31, which is specifically expressed on vascular endothelial cells; red channel) and CD105-targeted NPs (FITC-QD@HMSN(DOX)-PEG-TRC105; green channel), indicating the excellent targeting specificity. Due to their relatively large size, minimal extravasation of NPs from the tumor vasculature was observed, which emphasizes the importance of vasculature targeting over tumor cell targeting. On the other hand, non-targeted group (FITCQD@HMSN(DOX)-PEG) exhibited much lower tumor accumulation. Liver was selected as the positive control, since it is the major clearance organ of NPs. In our study, both targeted and non-targeted groups showed strong accumulation (green channel), and no correlation was found (a)
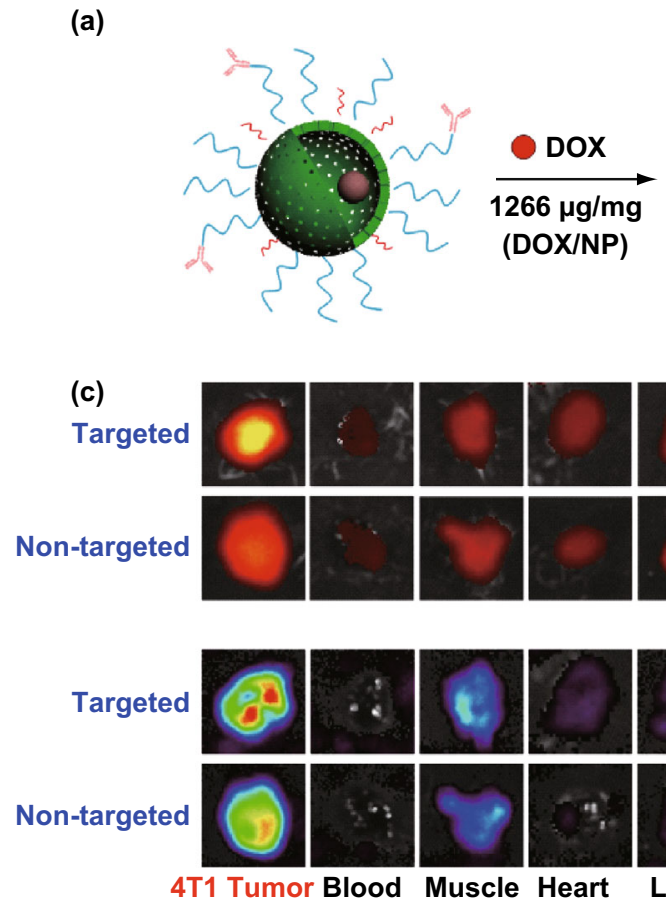

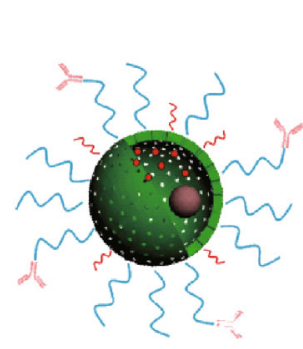

(b) $40[-\mathrm{QD} @ \mathrm{HMSN}(\mathrm{pH} 7.4)$

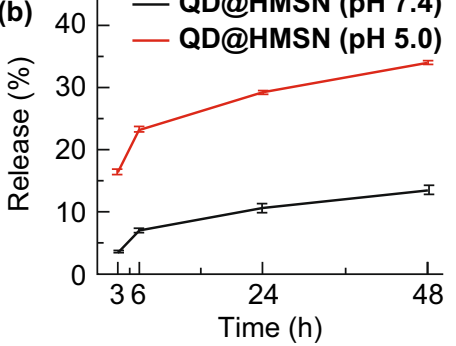

$7 \times 10^{6}$
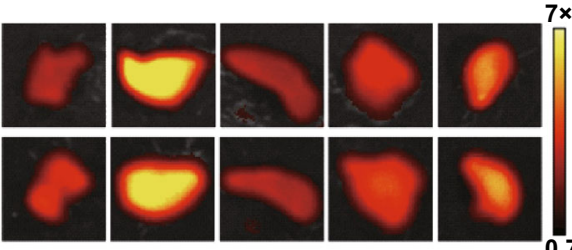

Signal from QD Ex: 605, Em: $700 \mathrm{~nm}$

Radiant efficiency (p/sec/cm $/$ sr $\}$

$\left(\mu \mathrm{W} / \mathrm{cm}^{2}\right)$
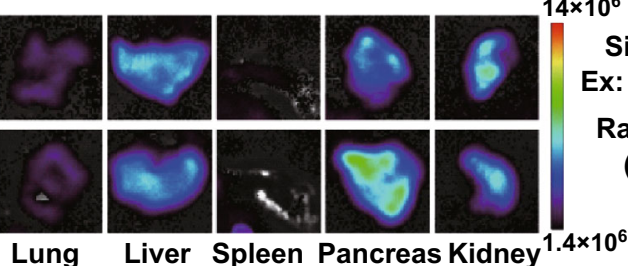

Signal from DOX Ex: 500, Em: $580 \mathrm{~nm}$

Radiant efficiency $\left(\mathbf{p} / \mathbf{s e c} / \mathrm{cm}^{2} / \mathbf{s r}\right\}$ $\left(\mu \mathrm{W} / \mathrm{cm}^{2}\right)$

Fig. 5 a A schematic illustration of DOX loading in QD@HMSN yolk/shell-structured silica nanosystem. b The drug release rates of QD@HMSN NPs at pH 7.4 and pH 5.0 at different incubation time points. c Fluorescence imaging of QD705 $\left(E_{x}: 605, E_{m}: 700 \mathrm{~nm}\right)$ and DOX $\left(E_{x}: 500, E_{\mathrm{m}}: 580 \mathrm{~nm}\right.$ ) from both NOTA-QD@HMSN(DOX)-PEG-TRC105 (targeted group) and NOTA-QD@HMSN(DOX)-PEG (non-targeted group) in $4 \mathrm{~T} 1$ tumor and other organs 


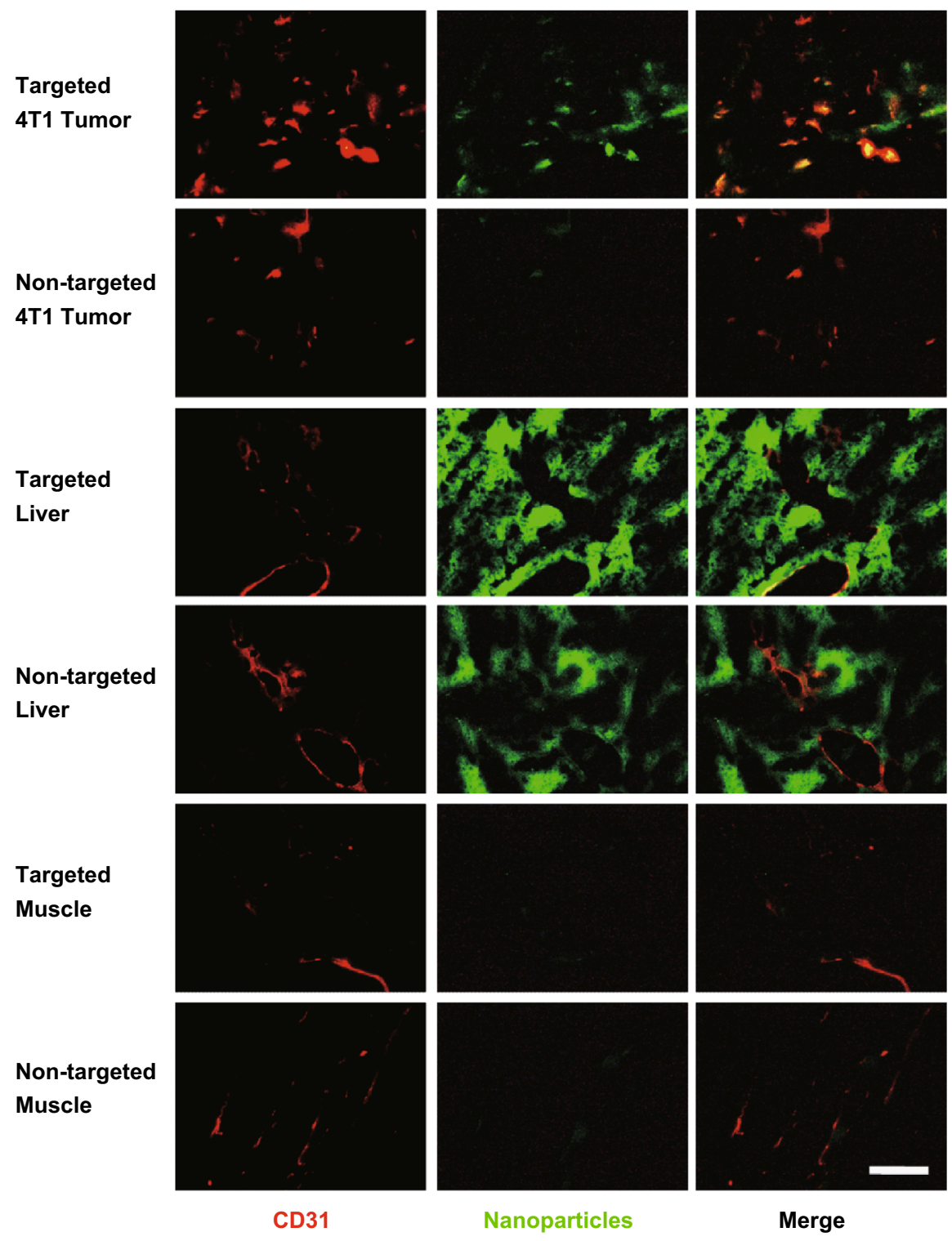

Fig. 6 Histological analysis of various tissue slices for CD31 (red, with anti-mouse CD31 primary antibody) and NPs (green, FITC-conjugated QD@HMSN NPs) from both FITC-NOTA-QD@HMSN-PEG-TRC105 (targeted group) and FITC-NOTA-QD@HMSN-PEG (non-targeted group). Scale bar: $100 \mu \mathrm{m}$. (Color figure online)

between the signals from vasculature and NPs in livers, suggesting that QD@HMSN NPs were captured by liver via nonspecific reticuloendothelial system (RES) uptake. In addition, minimal accumulation was found in muscle (negative control) in both targeted and non-targeted groups, which matched well with the results from PET imaging.

\section{Conclusion}

Herein, we report a radiolabeled antibody-conjugated yolk/ shell-structured silica nanosystem ${ }^{64} \mathrm{Cu}$-NOTAQD@HMSN-PEG-TRC105. Significant boost of tumor uptake (1.5-fold increase at early time points) and excellent imaging contrast was achieved with specific tumor vasculature targeting. Compared to conventional MSN, the greatly enhanced drug loading capacity $\left(1266 \mathrm{mg} \mathrm{g}^{-1}\right.$, DOX/NP weight ratio) was achieved with yolk/shell QD@HMSN NPs, which can be used for improved chemotherapy of cancer. Taking advantage of both imaging techniques, PET/optical dual-modality imaging was utilized to cross-validate the imaging results, providing a more reliable imaging outcome. By combining vasculature targeting, $\mathrm{pH}$-sensitive drug delivery and dual-modality imaging into a single platform, yolk/shell-structured silica nanosystem may be employed for the future image-guided 
tumor-targeted drug delivery, to further enhance the therapeutic efficacy and to enable cancer theranostics.

Acknowledgements This work was supported, in part, by the University of Wisconsin-Madison, the National Institutes of Health (P30CA014520 and T32CA009206), and the American Cancer Society (125246-RSG-13-099-01-CCE).

\section{Compliance with ethical standards}

Conflict of interest Charles P. Theuer is an employee of TRACON Pharmaceuticals, Inc. The other authors declare that they have no conflict of interest.

Open Access This article is distributed under the terms of the Creative Commons Attribution 4.0 International License (http://crea tivecommons.org/licenses/by/4.0/), which permits unrestricted use, distribution, and reproduction in any medium, provided you give appropriate credit to the original author(s) and the source, provide a link to the Creative Commons license, and indicate if changes were made.

\section{References}

1. H. Cabral, N. Nishiyama, K. Kataoka, Supramolecular nanodevices: from design validation to theranostic nanomedicine. Acc. Chem. Res. 44(10), 999-1008 (2011). https://doi.org/10.1021/ ar200094a

2. X. Ma, Y. Zhao, X.-J. Liang, Theranostic nanoparticles engineered for clinic and pharmaceutics. Acc. Chem. Res. 44(10), 1114-1122 (2011). https://doi.org/10.1021/ar2000056

3. W. Cai, X. Chen, Nanoplatforms for targeted molecular imaging in living subjects. Small 3(11), 1840-1854 (2007). https://doi.org/ 10.1002/smll.200700351

4. D.E. Lee, H. Koo, I.C. Sun, J.H. Ryu, K. Kim, I.C. Kwon, Multifunctional nanoparticles for multimodal imaging and theragnosis. Chem. Soc. Rev. 41(7), 2656-2672 (2012). https://doi. org/10.1039/c2cs15261d

5. J. Rieffel, U. Chitgupi, J.F. Lovell, Recent advances in higherorder, multimodal, biomedical imaging agents. Small 11(35), 4445-4461 (2015). https://doi.org/10.1002/smll.201500735

6. S. Shi, F. Chen, W. Cai, Biomedical applications of functionalized hollow mesoporous silica nanoparticles: focusing on molecular imaging. Nanomedicine 8(12), 2027-2039 (2013). https://doi.org/10.2217/nnm.13.177

7. J.L. Vivero-Escoto, R.C. Huxford-Phillips, W. Lin, Silica-based nanoprobes for biomedical imaging and theranostic applications. Chem. Soc. Rev. 41(7), 2673-2685 (2012). https://doi.org/10. $1039 / \mathrm{c} 2 \mathrm{cs} 15229 \mathrm{k}$

8. F. Tang, L. Li, D. Chen, Mesoporous silica nanoparticles: synthesis, biocompatibility and drug delivery. Adv. Mater. 24(12), 1504-1534 (2012). https://doi.org/10.1002/adma.201104763

9. X. Fang, X. Zhao, W. Fang, C. Chen, N. Zheng, Self-templating synthesis of hollow mesoporous silica and their applications in catalysis and drug delivery. Nanoscale 5(6), 2205-2218 (2013). https://doi.org/10.1039/c3nr34006f

10. S.H. Wu, C.Y. Mou, H.P. Lin, Synthesis of mesoporous silica nanoparticles. Chem. Soc. Rev. 42(9), 3862-3875 (2013). https:// doi.org/10.1039/c3cs35405a

11. F. Chen, H. Hong, S. Shi, S. Goel, H.F. Valdovinos, R. Hernandez, C.P. Theuer, T.E. Barnhart, W. Cai, Engineering of hollow mesoporous silica nanoparticles for remarkably enhanced tumor active targeting efficacy. Sci. Rep. 4, 5080 (2014). https:// doi.org/10.1038/srep05080

12. R. Chakravarty, S. Goel, H. Hong, F. Chen, H.F. Valdovinos, R. Hernandez, T.E. Barnhart, W. Cai, Hollow mesoporous silica nanoparticles for tumor vasculature targeting and pet imageguided drug delivery. Nanomedicine 10(8), 1233-1246 (2015). https://doi.org/10.2217/nnm.14.226

13. S. Goel, C.A. Ferreira, F. Chen, P.A. Ellison, C.M. Siamof, T.E. Barnhart, W. Cai, Activatable hybrid nanotheranostics for tetramodal imaging and synergistic photothermal/photodynamic therapy. Adv. Mater. 30(6), 1704367 (2018). https://doi.org/10. 1002/adma.201704367

14. J. Culver, W. Akers, S. Achilefu, Multimodality molecular imaging with combined optical and spect/pet modalities. J. Nucl. Med. 49(2), 169-172 (2008). https://doi.org/10.2967/jnumed.107. 043331

15. W. Cai, X. Chen, Multimodality molecular imaging of tumor angiogenesis. J. Nucl. Med. 49, 113S-128S (2008). https://doi. org/10.2967/jnumed.107.045922

16. S.N. Histed, M.L. Lindenberg, E. Mena, B. Turkbey, P.L. Choyke, K.A. Kurdziel, Review of functional/anatomical imaging in oncology. Nucl. Med. Commun. 33(4), 349-361 (2012). https://doi.org/10.1097/MNM.0b013e32834ec8a5

17. T.F. Massoud, S.S. Gambhir, Molecular imaging in living subjects: seeing fundamental biological processes in a new light. Genes Dev. 17(5), 545-580 (2003). https://doi.org/10.1101/gad. 1047403

18. R. Weissleder, M.J. Pittet, Imaging in the era of molecular oncology. Nature 452(7187), 580-589 (2008). https://doi.org/10. 1038/nature06917

19. S. Shi, C. Xu, K. Yang, S. Goel, H.F. Valdovinos et al., Chelatorfree radiolabeling of nanographene: breaking the stereotype of chelation. Angew. Chem. Int. Edit. 56(11), 2889-2892 (2017). https://doi.org/10.1002/anie.201610649

20. S. Goel, C.G. England, F. Chen, W. Cai, Positron emission tomography and nanotechnology: a dynamic duo for cancer theranostics. Adv. Drug Deliv. Rev. 113, 157-176 (2017). https:// doi.org/10.1016/j.addr.2016.08.001

21. K. Hu, H. Wang, G. Tang, T. Huang, X. Tang, X. Liang, S. Yao, D. Nie, In vivo cancer dual-targeting and dual-modality imaging with functionalized quantum dots. J. Nucl. Med. 56(8), 1278-1284 (2015). https://doi.org/10.2967/jnumed.115.158873

22. S. Shi, K. Yang, H. Hong, H.F. Valdovinos, T.R. Nayak et al., Tumor vasculature targeting and imaging in living mice with reduced graphene oxide. Biomaterials 34(12), 3002-3009 (2013). https://doi.org/10.1016/j.biomaterials.2013.01.047

23. B.K. Seon, A. Haba, F. Matsuno, N. Takahashi, M. Tsujie et al., Endoglin-targeted cancer therapy. Curr. Drug Deliv. 8(1), 135-143 (2011). https://doi.org/10.2174/156720111793663570

24. E. Fonsatti, H.J. Nicolay, M. Altomonte, A. Covre, M. Maio, Targeting cancer vasculature via endoglin/cd105: a novel antibody-based diagnostic and therapeutic strategy in solid tumours. Cardiovasc. Res. 86(1), 12-19 (2010). https://doi.org/10.1093/ cvr/cvp332

25. L.S. Rosen, M.S. Gordon, F. Robert, D.E. Matei, Endoglin for targeted cancer treatment. Curr. Oncol. Rep. 16(2), 365 (2014). https://doi.org/10.1007/s11912-013-0365-x

26. M. Paauwe, P. ten Dijke, L.J. Hawinkels, Endoglin for tumor imaging and targeted cancer therapy. Expert Opin. Ther. Targets 17(4), 421-435 (2013). https://doi.org/10.1517/14728222.2013. 758716

27. F. Chen, W. Bu, Y. Chen, Y. Fan, Q. He et al., A sub-50-nm monosized superparamagnetic $\mathrm{Fe}_{3} \mathrm{O}_{4} @ \mathrm{SiO}_{2}$ T2-weighted MRI contrast agent: highly reproducible synthesis of uniform singleloaded core-shell nanostructures. Chem. Asian J. 4(12), 1809-1816 (2009). https://doi.org/10.1002/asia.200900276 
28. K.M. Taylor, J.S. Kim, W.J. Rieter, H. An, W. Lin, Mesoporous silica nanospheres as highly efficient mri contrast agents. J. Am. Chem. Soc. 130(7), 2154-2155 (2008). https://doi.org/10.1021/ ja710193c

29. F. Chen, H. Hong, Y. Zhang, H.F. Valdovinos, S. Shi, G.S. Kwon, C.P. Theuer, T.E. Barnhart, W. Cai, In vivo tumor targeting and image-guided drug delivery with antibody-conjugated, radiolabeled mesoporous silica nanoparticles. ACS Nano 7(10), 9027-9039 (2013). https://doi.org/10.1021/nn403617j

30. S.P. Hadipour Moghaddam, M. Yazdimamaghani, H. Ghandehari, Glutathione-sensitive hollow mesoporous silica nanoparticles for controlled drug delivery. J. Control. Release (2018). https://doi.org/10.1016/j.jconrel.2018.04.032. (Epub ahead of print)

31. M. Kong, J. Tang, Q. Qiao, T. Wu, Y. Qi, S. Tan, X. Gao, Z. Zhang, Biodegradable hollow mesoporous silica nanoparticles for regulating tumor microenvironment and enhancing antitumor efficiency. Theranostics 7(13), 3276-3292 (2017). https://doi.org/ 10.7150/thno. 19987

32. M.S. Kang, R.K. Singh, T.H. Kim, J.H. Kim, K.D. Patel, H.W. Kim, Optical imaging and anticancer chemotherapy through carbon dot created hollow mesoporous silica nanoparticles. Acta Biomater. 55, 466-480 (2017). https://doi.org/10.1016/j.actbio. 2017.03.054 